\title{
Incorporating Texture Information into Polarimetric Radar Classification Using Neural Networks
}

\author{
Kaan Ersahin, Bernd Scheuchl and Ian Cumming \\ Department of Electrical and Computer Engineering \\ The University of British Columbia \\ Vancouver, BC, Canada
}

\begin{abstract}
Most of the recent research on polarimetric SAR classification focused on pixel-based techniques using the covariance matrix representation. Since multiple channels are inherently provided in polarimetric data, conventional techniques for increasing the dimensionality of the observation, such as texture feature extraction, were ignored. In this paper, we have demonstrated the potential of texture classification through gray level co-occurrence probabilities (GLCP), and proposed an unsupervised scheme using the self-organizing map (SOM) neural network. The increase in separability of the feature space is shown via the Fisher criterion and also verified by increased classification performance. Compared to the Wishart classifier, promising classification results are obtained from the Flevoland data set.
\end{abstract}

\section{INTRODUCTION}

Classification of polarimetric radar data, has been an important research topic for the last two decades. Improvements in understanding the properties of polarimetric data and increased computational capabilities over this period lead to new space missions for fully-polarized radar systems (RADARSAT-2 to be launched in late 2005). Researchers developed new techniques using multi-polarimetric data from airborne platforms, such as AIRSAR of JPL, trying to find better ways to interpret this rich source of information.

Since polarimetric data is often multi-looked for speckle reduction or compression, statistical characteristics of such data was studied extensively and based on the findings, Lee $e t$ al. [1] used the Wishart distribution to describe the statistics of the covariance matrix, allowing a maximum likelihood classification scheme to be developed. Since then, a lot of effort has been devoted to improve the performance based on this concept, which is known to utilize the complete polarimetric information. Cloude's decomposition was later used to provide the basis for direct classification of polarimetric data [2], [3].

A recent study [4] on classification of agricultural fields over Flevoland argues that the statistics for a collection of homogeneous areas of a class is not necessarily well described by the Wishart distribution. This limitation is mainly due to the a-priori assumption of a particular metric, a distance measure in the multidimensional feature space. Unless the feature space is separable by this metric, the classifier will perform poorly.

This work is supported by an NSERC-CRD grant, and by the BC Advanced Systems Institute.
Since neural network approaches do not make such an assumption, but instead determine this metric during the training procedure, they can perform better in such cases [5]. In this study, the self-organizing map (SOM) is used for performing the classification task [6].

Another motivation is that most of the work in the area focused on pixel-based classification using the covariance matrix elements and ignored the spatial information. Given the complex nature of the problem, we consider feature extraction to be an important part of this task and propose to employ gray level co-occurrence probabilities (GLCP) for texture feature extraction. These features, when used in addition to the covariance matrix parameters, are expected to improve the classification accuracy.

\section{STUdy AREA AND DATA Used}

A multi-look processed L-band AIRSAR data over Flevoland is used in this study. The ground truth is available for 13 classes, as shown in Figure 1. From each class, 200 samples were randomly chosen and divided into training and test sets.

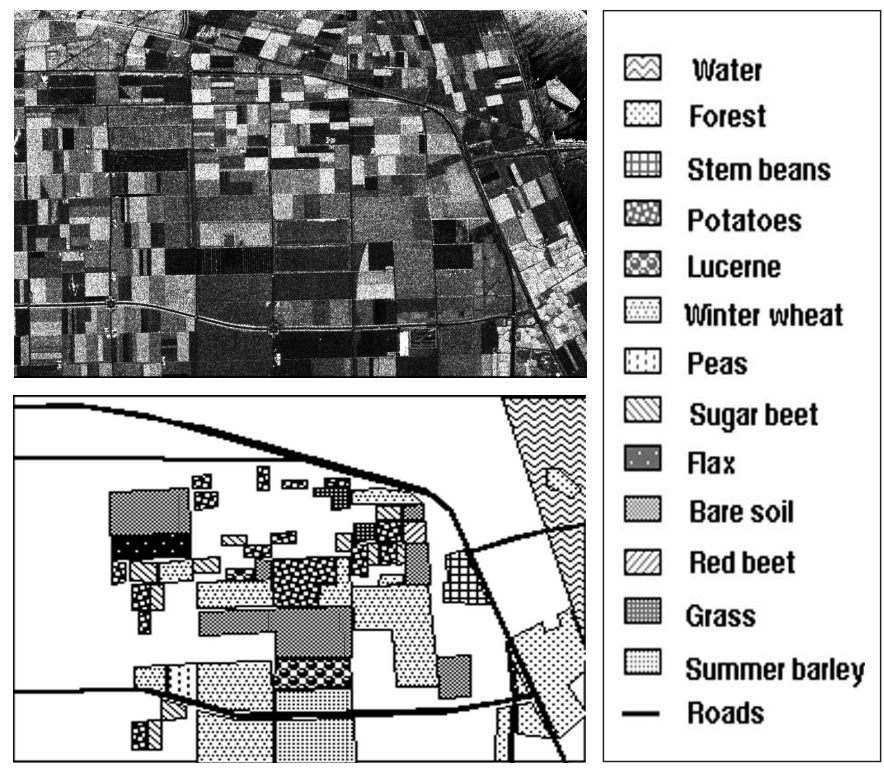

Fig. 1. L-band AIRSAR data and the ground truth of Flevoland 


\section{Texture Feature EXtraction}

The gray level co-occurrence probabilities (GLCP) provide second-order statistics for generating texture features. These represent conditional joint probabilities of all pair wise combinations of pixel values in the window of interest. For each pixel pair, two parameters describe the geometric relationship: interpixel distance $(\delta)$ and orientation $(\theta)$. The co-occurrence probability between gray level $i$ and $j$, is given by

$$
P_{i j} \mid(\delta, \theta)=\frac{N_{i j}}{\sum_{i, j=1}^{G} N_{i j}}
$$

where $N_{i j}$ represents the number of occurrences of gray levels $i$ and $j$ within a window, given a certain $(\delta, \theta)$ pair and $G$ is the number of quantized gray levels of the radar measurement (e.g. covariance matrix elements)

\section{A. Selection of Texture Statistics}

Texture measures appropriate for SAR imagery are studied in [7] which can be categorized in three groups: Smoothness Statistics, Homogeneity Statistics and Correlation. Selecting only one from each group is recommended, since they are well correlated. Following these discussions, three texture measures are selected:

$$
\begin{array}{cl}
\text { Contrast } & \sum P_{i j}(i-j)^{2} \\
\text { Entropy } & \sum P_{i j} \log \left(P_{i j}\right) \\
\text { Correlation } & \sum \frac{\left(i-\mu_{x}\right)\left(j-\mu_{y}\right) P_{i j}}{\sigma_{x} \sigma_{y}}
\end{array}
$$

The definition for contrast given by [8] is the difference between the highest and lowest values of a contiguous set of pixels. Entropy is an homogeneity measure, showing high values when the image is not texturally uniform and correlation is expressed by the correlation coefficient between two random variables, the pixel pair, in this case.

\section{B. Selection of $G, \delta$ and $\theta$}

The quantization level, $\mathrm{G}$, has various effects on different texture measures. Although it is expected that finer quantization would increase the classification accuracy, Clausi [7] concludes that it is only true for contrast and correlation. In the case of entropy, high values of $G$ causes fewer repeating pairs in the grey level co-occurrence matrix (GLCM), resulting in entropy values closer to 1 , as a result separability and classification accuracy is reduced. The discussion in the literature on the choice of interpixel distance, $\delta$, recommends to use 2 [9], [10]. On the other hand, four possible settings for orientation, $\theta,(0,45,90,135$ degrees), can be used to discriminate between orientations of features (e.g. lines) in an image. But if one needs rotation invariant features, averaging over orientation is appropriate.

\section{Selection of the Window Size}

Window size is a trade-off between classification accuracy, and boundary confusion, since using larger window will give better results for homogenous areas, but fail to detect the boundaries when there is mixture of classes. To choose an optimal window size calculating the coefficient of variation $(C V)$ in relation to window size is suggested by [11], where the objective is keeping the window size small and choosing the value where the CV starts to stabilize.

\section{Evaluation of the Extracted Features}

The Fisher criterion [12], is used to evaluate the features in terms of the class separability they provide. This measure calculates a ratio of between class separability and within-class variation. Higher values stands for improved separability, thus better classification performance is expected.

\section{Classification Using the Self-Organizing MaP}

\section{A. Training of the SOM}

A Self-Organizing Map (SOM) is an unsupervised technique in which the multi-dimensional feature space is mapped onto a 2D output map and the topology preserves the information gathered from all the input patterns. Unlike most other neural network structures, the principle of winner-takes-all is used. For any input, all neurons compete in terms of their distance to the input vector and only one output neuron becomes activated, called the winning neuron. SOM does not try to minimize an error function based on the expected output, instead the weights of the winning neuron and its neighborhood change according to (6).

$$
w_{i j}(t+1)=w_{i j}(t)+\alpha(t) \gamma(t)\left(x_{j}-w_{i j}(t)\right)
$$

where $w_{i j}(t+1)$ is the winning neuron, $x_{j}$ is the input vector, $\alpha(t)$ is the learning rate, and $\gamma(t)$ represents the neighborhood function. $\alpha(t)$ and $\gamma(t)$ both decay over time.

The training procedure can be explained as follows:

1. Normalize the input vectors for all samples

2. Initialize the weights with randomly selected input vectors

3. Calculate the activation of each node for the input vector

4. Find the winning neuron (the one with the best activation)

5. Adjust the weights according to (5)

6 . Repeat steps 3 - 5 for all the samples

7. Repeat step 6 for the required number of epochs

\section{B. Labeling of the Neurons}

After the training is complete, the map has been organized preserving the topology of the data, but the boundaries between classes are not yet defined. Since a classification task will be performed, each output node of the trained map needs to be assigned to a class, so that when new inputs are presented, the network will be able to make a decision.

The manual labeling requires the class information of the training samples, but the only reason to include this 
information is to create boundaries in the map and assign a class to each output node where new input vectors could later be classified. Therefore the predetermined classification of the training set will not bias the training process because it is used only at the end of the training [13].

\section{The Proposed Algorithm And Results}

\section{A. Speckle Reduction}

Most of the times speckle reduction is beneficial for classification, but for multi-polarized data, it is important to preserve the polarimetric properties. Therefore, the speckle filter suggested by Lee et al. [14], is chosen and the window size is set to $5 \times 5$ to preserve the texture information as recommended.

\section{B. Texture Feature Extraction}

As indicated in Section III.A, three texture features, contrast, entropy and correlation, are calculated from the GLCP. Following the discussions in Section III.B, $\delta=2$ is used and the gray level quantization $(G)$ is set to 32 as a compromise between optimum values for entropy and contrast. Since discrimination between different orientations is not our concern, we used averaging to get rotation invariant features. For the purpose of window size selection, the coefficient of variation (CV) for each class is calculated using window sizes from $5 \times 5$ to $19 \times 19$ to find where CV stabilizes. The optimum window size was found to vary between $9 \times 9$ to $13 \times 13$ for different input features.

\section{Evaluation of the Features}

The Fisher criterion is used to compare different set of features in terms of cluster separability. These features are extracted from inputs, such as the nine independent parameters from the covariance matrix (Cov9), the eigenvalues $\left(\lambda_{1} / \lambda_{2} / \lambda_{3}\right)$ or entropy, anisotropy, alpha angle (H/A/ $\alpha$ ) parameters following the procedure shown in Figure 2.

The conclusion is that the separability increases with the use of textural features, which shows promise to utilize this information for classification. In addition, filtering is found to increase the performance of pixel values, but decreases the separability of texture measures, since textural information is reduced together with the speckle. These graphs are not shown due to space limitations.

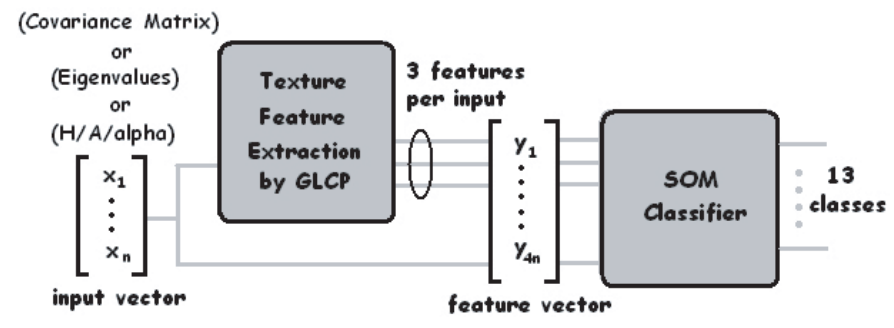

Fig. 2. Proposed scheme for polarimetric SAR classification

\section{Classification Results using SOM}

A SOM neural network is employed for the classification task and the results are obtained for feature vectors from a variety of inputs and window size settings for GLCM calculation. The graphs of accuracy vs. window size were used to verify the optimum size, and it was found that changing the window size to a larger value than $13 \times 13$ does not affect the accuracy a lot. These results agree with the the window size found by the coefficient of variation and seem to be an optimum choice for the selected data samples. On the other hand, using large window sizes will cause confusion near boundaries, which should be noted as a drawback for segmentation.

Table 1 shows the classification accuracy values averaged over a number of trials for the $13 \times 13$ window size. The choice of features and filtering results agree with the Fisher criterion and show that speckle filtering increases the classification accuracy only when pixel values are used. The same applies to contrast, but not to entropy and correlation. In these cases the accuracy is decreased due to the reduction of texture information. Similarly when all three texture features are used, the accuracy is higher for the original unfiltered data.

TABLE I

Overall Classification AcCuracy AChieved Using the Proposed CLASSIFICATION SCHEME

\begin{tabular}{llcccc} 
Filter & Features & $\mathrm{H} / \mathrm{A} / \alpha$ & $\lambda_{1} / \lambda_{2} / \lambda_{3}$ & Cov9* & Cov5** \\
\hline None & Pixel value & 16.9 & 26.6 & 22.6 & 29.2 \\
& Correlation & 13.5 & 19.1 & 21.5 & 20.7 \\
& Entropy & 34.0 & 60.7 & 73.9 & 68.0 \\
& Contrast & 37.4 & 62.9 & 65.1 & 70.7 \\
& Corr/Entr/Cont & 34.3 & 60.3 & 72.9 & 67.3 \\
& All 4 & 35.3 & 61.0 & 71.9 & 68.1 \\
\hline $5 \times 5$ & Pixel value & 44.0 & 52.2 & 46.0 & 59.5 \\
& Correlation & 14.2 & 25.7 & 25.2 & 24.9 \\
& Entropy & 31.3 & 50.2 & 47.5 & 61.1 \\
& Contrast & 38.9 & 59.5 & 70.7 & 70.2 \\
& Corr/Entr/Cont & 32.5 & 54.3 & 47.5 & 62.2 \\
& All 4 & 32.8 & 55.6 & 47.0 & 61.2 \\
\hline
\end{tabular}

$*|H H|^{2},|H V|^{2},|V V|^{2}, \rho_{H H V V}, \rho_{H V V V}, \rho_{H V H H}, \theta_{H H V V}, \theta_{H V V V}, \theta_{H V H H}$ $* *|H H|^{2},|H V|^{2},|V V|^{2}, \rho_{H H V V}, \theta_{H H V V}$

The results obtained without filtering show that when covariance matrix is used as the input to the proposed scheme, accuracies in the range of $65.1 \%$ to $73.9 \%$ are obtained. If filtered data is used, the highest value $(70.7 \%)$ comes from contrast of Cov9*. The confusion matrix for unfiltered data classified by the proposed scheme using Correlation/Entropy/Contrast features of Cov9* is given in Table 2.

For the purpose of comparison, the Bayesian classifier based on the Wishart distribution is used for the same task resulting in the confusion matrix given in Table 3 with an overall accuracy of $65.54 \%$. If compared to the obtained results in Table 1, we can argue that the proposed scheme results in similar or higher accuracies when the covariance matrix parameters are utilized. When the eigenvalues are used, 62.84 $\%$ accuracy is achieved. The use of H/A/alpha for texture feature extraction is not recommended since the accuracy compared to the pixel-based classification is decreased. 
TABLE II

Confusion Matrix for the Proposed Scheme Using Correlation/Entropy/Contrast of the UnFILTEREd Cov9* Parameters

\begin{tabular}{|c|c|c|c|c|c|c|c|c|c|c|c|c|c|}
\hline & Water & Forest & Stem Beans & Potatoes & Lucerne & W. Wheat & Peas & Sugar Beet & Flax & Bare Soil & Red Beet & Grass & S. Barley \\
\hline Water & 94 & 0 & 0 & 1 & 0 & 0 & 0 & 3 & 2 & 1 & 0 & 0 & 0 \\
\hline Forest & 4 & 94 & 0 & 2 & 0 & 0 & 0 & 0 & 0 & 0 & 0 & 0 & 0 \\
\hline Stem Beans & 4 & 0 & 96 & 0 & 0 & 0 & 0 & 0 & 0 & 0 & 0 & 0 & 0 \\
\hline Potatoes & 2 & 1 & 0 & 72 & 0 & 3 & 1 & 12 & 0 & 2 & 4 & 2 & 3 \\
\hline Lucerne & 5 & 0 & 1 & 1 & 90 & 3 & 0 & 0 & 0 & 0 & 0 & 0 & 1 \\
\hline W. Wheat & 5 & 0 & 1 & 2 & 0 & 54 & 1 & 5 & 1 & 14 & 2 & 4 & 12 \\
\hline Peas & 0 & 0 & 0 & 0 & 0 & 0 & 100 & 0 & 0 & 0 & 0 & 0 & 0 \\
\hline Sugar Beet & 1 & 1 & 0 & 19 & 0 & 5 & 0 & 35 & 0 & 1 & 35 & 0 & 2 \\
\hline Flax & 3 & 0 & 0 & 0 & 0 & 0 & 0 & 0 & 97 & 0 & 0 & 0 & 0 \\
\hline Bare Soil & 1 & 0 & 0 & 4 & 0 & 11 & 14 & 2 & 1 & 40 & 2 & 7 & 19 \\
\hline Red Beet & 2 & 0 & 0 & 1 & 0 & 5 & 0 & 11 & 0 & 3 & 73 & 0 & 5 \\
\hline Grass & 0 & 0 & 0 & 3 & 0 & 3 & 0 & 3 & 1 & 13 & 4 & 62 & 11 \\
\hline S. Barley & 1 & 0 & 0 & 5 & 0 & 22 & 3 & 1 & 0 & 8 & 0 & 19 & 41 \\
\hline
\end{tabular}

$*|H H|^{2},|H V|^{2},|V V|^{2}, \rho_{H H V V}, \rho_{H V V V}, \rho_{H V H H}, \theta_{H H V V}, \theta_{H V V V}, \theta_{H V H H}$ Overall accuracy $=72.9$

TABLE III

Confusion Matrix For the Wishart Classifier Using the Covariance Matrix After Speckle Filtering With a Window Size of 7 X7

\begin{tabular}{|c|c|c|c|c|c|c|c|c|c|c|c|c|c|}
\hline & Water & Forest & Stem Beans & Potatoes & Lucerne & W. Wheat & Peas & Sugar Beet & Flax & Bare Soil & Red Beet & Grass & S. Barley \\
\hline Water & 49 & 0 & 0 & 1 & 0 & 1 & 2 & 0 & 45 & 1 & 0 & 1 & 0 \\
\hline Forest & 0 & 79 & 0 & 13 & 3 & 0 & 0 & 1 & 0 & 0 & 3 & 0 & 1 \\
\hline Stem Beans & 0 & 2 & 97 & 1 & 0 & 0 & 0 & 0 & 0 & 0 & 0 & 0 & 0 \\
\hline Potatoes & 0 & 20 & 0 & 53 & 0 & 6 & 0 & 6 & 0 & 1 & 13 & 0 & 1 \\
\hline Lucerne & 0 & 1 & 0 & 0 & 98 & 0 & 0 & 0 & 0 & 2 & 0 & 0 & 0 \\
\hline W. Wheat & 0 & 0 & 0 & 1 & 2 & 34 & 8 & 10 & 0 & 9 & 2 & 3 & 31 \\
\hline Peas & 0 & 0 & 0 & 0 & 0 & 0 & 99 & 1 & 0 & 0 & 0 & 0 & 0 \\
\hline Sugar Beet & 0 & 2 & 0 & 18 & 0 & 0 & 2 & 54 & 0 & 1 & 23 & 0 & 0 \\
\hline Flax & 2 & 0 & 0 & 0 & 0 & 0 & 0 & 0 & 98 & 0 & 0 & 0 & 0 \\
\hline Bare Soil & 0 & 0 & 0 & 0 & 1 & 16 & 12 & 3 & 0 & 52 & 0 & 6 & 10 \\
\hline Red Beet & 0 & 0 & 0 & 0 & 0 & 0 & 2 & 5 & 0 & 0 & 59 & 34 & 0 \\
\hline Grass & 0 & 0 & 0 & 0 & 11 & 10 & 0 & 0 & 1 & 0 & 0 & 71 & 7 \\
\hline S. Barley & 0 & 0 & 0 & 0 & 0 & 19 & 7 & 0 & 0 & 9 & 0 & 56 & 9 \\
\hline
\end{tabular}

$*|H H|^{2},|H V|^{2},|V V|^{2}, \rho_{H H V V}, \rho_{H V V V}, \rho_{H V H H}, \theta_{H H V V}, \theta_{H V V V}, \theta_{H V H H}$

Overall accuracy $=65.5$

\section{CONCLUSION}

In this paper, we have demonstrated the potential of using texture features obtained from the gray level co-occurrence probabilities (GLCP) for the classification of polarimetric SAR data. The increase in separability of the feature space is shown using the Fisher criterion and also verified with the classification accuracies obtained. The self-organizing map (SOM) is used for classification and promising results were obtained for the sample data set compared to the Wishart classifier. Further validation with larger data sets is ongoing.

\section{ACKNOWLEDGMENT}

The authors would like to thank L. Ferro-Famil for providing the AIRSAR data and ground truth of Flevoland.

\section{REFERENCES}

[1] J. S. Lee, M. R. Grunes and R. Kwok, Classification of Multi-look Polarimetric SAR Imagery based on Complex Wishart Distribution, Int. J. of Remote Sensing, vol.15, no.11, p.2299-2311, 1994.

[2] L. Ferro-Famil, E. Pottier and J. S. Lee, Unsupervised Classification of Multi-frequency and Fully Polarimetric SAR Images Based on the H/A/Alpha - Wishart Classifier, IEEE Trans. on Geoscience and Remote Sensing, vol.39, no.11, p.2332-2342, 2001.

[3] E. Pottier and J. S. Lee, Application of the $\langle H / A / \alpha>$ Polarimetric Decomposition Theorem For Unsupervised Classification of Fully Polarimetric SAR Data Based on the Wishart Distribution, European Space Agency (Special Publication) ESA SP, n SP-450, p.335-340, Mar 2000.
[4] D. H. Hoekman and M. A. M. Vissers, A New Polarimetric Classification Approach Evaluated for Agricultural Crops, IEEE Trans. on Geoscience and Remote Sensing, vol.41, no.12, p.2881-2889, 2003.

[5] J. A. Benediktsson, P. H. Swain, O. K. Ersoy, Neural Network Approaches Versus Statistical Methods In Classification Of Multisource Remote Sensing Data, IEEE Trans. on Geoscience and Remote Sensing, vol.28, no.4, p.540-552, 1990.

[6] T. Kohonen, Self-Organizing Maps Springer Series in Information Sciences, Vol. 30, Springer, 2001.

[7] D. A. Clausi, An Analysis of Co-occurrance Texture Statistics as a Function of Grey Level Quantization, Canadian J. Remote Sensing, vol.28, no.1, p.45-62, 2002.

[8] A. Baraldi and F. Parmigianni, An Investigation of the Textural Characteristic Associated with Gray Level Cooccurrence Matrix Statistical Parameters, IEEE Trans. on Geoscience and Remote Sensing, vol.33, no.2, p.293-304, 1995.

[9] Q. A. Holmes, D. R. Nuesch and R. A. Schuchman, Textural Analysis and Real-time Classification of Sea-Ice Types using Digital SAR Data, IEEE Trans. on Geoscience and Remote Sensing, vol.22, no.2, p.113-120, 1984.

[10] M. E. Shokr, Evaluation of Second-order Texture Parameters for Sea Ice Classification from Radar Images, J. Geophysical Research, vol.96, n.C6, p.10625-10640, 1991.

[11] H. Anys and D. C. He, Evaluation of Textural and Multipolarization Radar Features for Crop Classification, IEEE Trans. on Geoscience and Remote Sensing, vol33., no.5, p.1170-1181, 1995.

[12] R. H. Duda, P. E. Hart and D. G. Stork, Pattern Classification, $2^{\text {nd }}$ ed., New York: John Wiley and Sons Inc., p.664, 2001.

[13] B. L. Vrusias, Combining Unsupervised Classifiers: A Multimodal Case Study, PhD Dissertation, University of Surrey, Guildford, Surrey, 2004.

[14] J. S. Lee, M. R. Grunes and G. de Grandi, Polarimetric SAR Speckle Filtering and It's Implications for Classification, IEEE Trans. on Geoscience and Remote Sensing, vol.37, no.5, p.2363-2373, 1999. 